\title{
Probióticos na modulação intestinal como adjuvante no tratamento de sinais e sintomas de depressão
}

\author{
Probiotics in intestinal modulation as an adjuvant in the treatment of signs and symptoms of \\ depression
}

Probióticos en modulación intestinal como adyuvante en el tratamiento de signos y síntomas

depresión

Recebido: 29/05/2021 | Revisado: 19/06/2021 | Aceito: 07/07/2021 | Publicado: 09/07/2021

Erick Michell Bezerra Oliveira

ORCID: https://orcid.org/0000-0001-6056-2863

Hospital Macro Regional de Caxias, Brasil

E-mail: erickmichell1@hotmail.com

Manoel Augusto de Moura

ORCID: https://orcid.org/0000-0001-5721-5780

UniFacema, Brasil

E-mail: coachingmoura@gmail.com

Luanna da Silva de Assunção

ORCID: https://orcid.org/0000-0003-3767-3447

Hospital Macro Regional de Caxias, Brasil

E-mail: luannacx2023@gmail.com

Aline Cristina Ribeiro da Luz

ORCID: https://orcid.org/0000-0001-6377-9312

Unidade de Pronto Atendimento de Coroatá, Brasil

E-mail: alineclfisio123@gmail.com

Adyelle Karoline Atan

ORCID: https://orcid.org/0000-0001-6708-3021

UniFacema, Brasil

E-mail: adyelle.atan12@gmail.com

Rubenilson Luna Matos

ORCID: https://orcid.org/0000-0002-4744-9396

Coordenador da Atenção Primária de Caxias, Brasil

E-mail: rubenilsonluna@ hotmail.com

Julianne de Area Leão Pereira da Silva

ORCID: https://orcid.org/0000-0003-4529-0104

Hospital de Campanha de Caxias, Brasil

E-mail: juliannealp@yahoo.com.br

Thiago Henrique Gonçalves Moreira

ORCID: https://orcid.org/0000-0002-2355-3148

Coordenador da Saúde Bucal de Caxias, Brasil

E-mail: drthiagohenrique@hotmail.com

Jonas Alves Cardoso

ORCID: https://orcid.org/0000-0002-5568-2024

Hospital Macro Regional de Caxias, Brasil

E-mail: jonasalves@pi.senac.br

Flávio Bruno Rodrigues de Assunção

ORCID: https://orcid.org/0000-0002-5982-7542

Hospital de Campanha de Caxias, Brasil

E-mail: f-bruno69@hotmail.com

Domingas Marlyene Silva Conceição

ORCID: https://orcid.org/0000-0003-2189-5344

Hospital Macro Regional de Caxias, Brasil

E-mail: marllyene1212@gmail.com

Francisco de Assis Viana dos Santos

ORCID: https://orcid.org/0000-0003-3047-260X

Hospital Macro Regional de Caxias, Brasil

E-mail: assissantosf9@gmail.com

Géofrey Henrick Silva Galvão

ORCID: https://orcid.org/0000-0002-6668-8624

Hospital Geral Municipal de Codó, Brasil

E-mail: herick_102@hotmail.com

Hernandys Ribeiro Bezerra

ORCID: https://orcid.org/0000-0001-7261-3920

Hospital de Campanha de Caxias, Brasil

E-mail: hernandys01@hotmail.com 


\author{
Nathália Cássia Martins Sampaio \\ ORCID: https://orcid.org/0000-0003-4475-4771 \\ Hospital de Campanha de Caxias, Brasil \\ E-mail: nathaliasampaiofisio@gmail.com
}

\begin{abstract}
Resumo
Objetivo: Este estudo teve como objetivo investigar se os probióticos melhoraram sintomas de depressão e ansiedade em avaliadas por diferentes escalas psicométricas. Metodologia: $O$ presente estudo trata-se de uma revisão bibliográfica do tipo integrativa, realizada por meio de um levantamento de literatura pesquisa bibliográfica no PubMed combinando os termos "Probióticos", "Modulação Intestinal" e "Depressão" para identificar os estudos de ensaios clínicos em humanos que investigaram as relações entre aspectos depressivos, ansiosos e a microbiota. Resultados: Os dados foram extraídos de 12 estudos de ensaios clínicos (922 humanos), randomizado, controlados, duplo-cego. No contexto avaliado, probióticos reduziram pontuação nas diferentes escalas psicométricas reduzindo o comportamento semelhante a ansiedade e depressivo. Análises de análises em nível de espécie probiótica, identificaram subespécies do gênero Lactobacillus e Bifidobacterium a capacidade de atividade ansiolítica. Melhoras $\geqslant 60 \%$ em escalas avaliativas foram demonstradas com suplementação de probióticos e até mesmo melhoras na qualidade de vida em indivíduos com doenças inflamatórias intestinais. Conclusão: A microbiota foi alçada a um alvo potencial para o tratamento de transtornos psiquiátricos e antidepressivos. A depressão é um desafio para a saúde global, pois demanda intervenções multidisciplinar já que está doença se caracteriza como uma desordem metabólica cerebral e os probióticos desempenhariam uma ação essencial sobre o estado de saúde e doença do indivíduo.
\end{abstract}

Palavras-chave: Depressão; Probióticos; Microbiota.

\begin{abstract}
Objective: This study aimed to investigate whether probiotics improved symptoms of depression and anxiety as assessed by different psychometric scales. Methodology: The present study is an integrative bibliographic review, carried out through a literature survey bibliographic research in PubMed combining the terms "Probiotics", "Intestinal Modulation" and "Depression" to identify clinical trial studies in humans who investigated the relationship between depressive, anxious aspects and the microbiota. Results: Data were extracted from 12 studies of clinical trials (922 humans), randomized, controlled, double-blind. In the assessed context, probiotics reduced scores on different psychometric scales, reducing anxiety-like and depressive behavior. Analyzes of analyzes at the level of probiotic species, identified subspecies of the genus Lactobacillus and Bifidobacterium the capacity for anxiolytic activity. $\geqslant 60 \%$ improvements in evaluation scales were demonstrated with supplementation of probiotics and even improvements in quality of life in individuals with inflammatory bowel diseases. Conclusion: The microbiota was raised to a potential target for the treatment of psychiatric and antidepressant disorders. Depression is a challenge for global health, as it requires multidisciplinary interventions since this disease is characterized as a cerebral metabolic disorder and probiotics would play an essential role on the individual's health and disease status.
\end{abstract}

Keywords: Depression; Probiotics; Microbiota.

\title{
Resumen
}

Objetivo: Este estudio tuvo como objetivo investigar si los probióticos mejoraron los síntomas de depresión y ansiedad evaluados por diferentes escalas psicométricas. Metodología: El presente estudio es una revisión bibliográfica integrativa, realizada mediante una encuesta bibliográfica de investigación bibliográfica en PubMed combinando los términos "Probióticos", "Modulación intestinal" y "Depresión" para identificar estudios de ensayos clínicos en humanos que investigaron la relación entre aspectos depresivos, ansiosos y la microbiota. Resultados: Se extrajeron datos de 12 estudios de ensayos clínicos (922 humanos), aleatorizados, controlados, doble ciego. En el contexto evaluado, los probióticos redujeron las puntuaciones en diferentes escalas psicométricas, reduciendo el comportamiento depresivo y de ansiedad. Análisis de análisis a nivel de especies probióticas, identificaron subespecies del género Lactobacillus y Bifidobacterium la capacidad de actividad ansiolítica. Se han demostrado mejoras de aproximadamente un $60 \%$ en las escalas de evaluación con la suplementación de probióticos e incluso mejoras en la calidad de vida en personas con enfermedades inflamatorias del intestino. Conclusión: La microbiota se convirtió en un objetivo potencial para el tratamiento de trastornos psiquiátricos y antidepresivos. La depresión es un desafío para la salud global, ya que requiere intervenciones multidisciplinarias ya que esta enfermedad se caracteriza como un trastorno metabólico del cerebro y los probióticos jugarían un papel fundamental en la salud y enfermedad del individuo.

Palabras clave: Depresión; Probióticos; Microbiota.

\section{Introdução}

A Organização Mundial da Saúde (OMS), afirma que depressão é uma desordem mental que afeta cerca de 322 milhões de pessoas em todo mundo sendo uma doença incapacitante e uma das principais contribuintes para a carga global de 
doenças com forte prevalência em homens e mulheres, mas afeta especialmente as mulheres. Depressão é uma desordem mental com prevalência ao longo da vida de $7 \%$ a $21 \%$ e com comprometimento substancial da funcionalidade do indivíduo, com redução da qualidade de vida do portador e do cuidador, e com maior risco de mortalidade a seus portadores (Bromet et al 2011).

Dados do Ministério da Saúde (2019) apontam que a prevalência da depressão no Brasil está em torno de 15,8\% com maior incidência na terceira década de vida, mas pode se manifestar em qualquer faixa etária, e ao longo da vida apresenta prevalência de $20 \%$ para mulheres é $12 \%$ para homens. O Ministério da Saúde caracteriza a depressão em três grupos distintos: 1) genético, com $40 \%$ de suscetibilidade; 2) Bioquímica Cerebral, alterações em neurotransmissores noradrenalina, serotonina e dopamina; 3) Eventos vitais, exposição a eventos estressores em indivíduos suscetíveis a manifestar a doença.

A influência genética na desordem depressiva é comprovada por estudos realizados em indivíduos gêmeos. Estudos genéticos moleculares que incluem estudos por associação em todo genoma, apenas um pequeno grupo de genes foram identificados como candidatos na causa genética da patogênese da depressão. Os estudos denominam o fator genético como "herdabilidade", com uma estimativa que pode variar de $37 \%$ até $70 \%$ considerando fatores como idade, recaídas depressivas e a gravidade da manifestação forem consideradas. Os fatores epigenéticos estão associados aos mecanismos de metilação do DNA (Menke et al., 2012).

A depressão além de uma doença psiquiátrica recorrente e que desabilita seu portador de interações sociais e com impactos até mesmo na vida econômica e sobre sistemas públicos de saúde. Estima-se que cerca de 300 milhões de indivíduos sejam portadores de depressão e associados a cerca de 800 mil suicídios anuais (WHO, 2017). Apesar do fato de medicações antidepressivas serem amplamente reconhecidas como uma opção de tratamento, uma parcela significativa de pacientes parece não responder a estas estratégias farmacológicas (Gelenberg et al., 2010) Cerca de 50\% de pacientes diagnosticados com disbiose, diminuição da barreira intestinal associada a inflamações, são também diagnosticados com depressão (Bhandari et al., 2017). Como resultado da disbiose, prevalece uma baixa capacidade de metabolismo de carboidratos e alta capacidade de metabolismo proteicos, pode ser associada a sintomatologia da depressão por uma deficiência energética e baixa produção de ácidos graxos de cadeia curta (AGCC) (Bhandari et al., 2017). Em quadro de disbiose a baixa produção de micronutrientes associados a microbiota, pode ser agravada pela escolha errônea de alimentos em indivíduos já depressivos agravando a fisiopatologia da doença (Kwak et al., 2016).

Nesse sentido a microbiota intestinal tem sido associada como o principal regulador na comunicação bidirecional entre cérebro e intestino, eixo intestino-cérebro (Sandhu et al., 2017). Uma série de estudos clínicos evidenciam a que o genoma produzido pela microbiota, pode ser um fator determinante na etiologia de doenças neurodegenerativas e no desenvolvimento neuronal. Estudos conduzidos em animais, o transplante de microbiota pode transferir o fenótipo comportamental associando a microbiota intestinal como agente modificador de comportamento e como chave inicial para $o$ desenvolvimento de desordens neuropsiquiátricas (Cenit et al., 2017).

A comunicação bilateral no eixo intestino-cérebro ocorre através do nervo vago, vias hormonais, neuroendócrinas e imunológicas. A associação da microbiota intestinal e hipotálamo-pituitária-adrenal (HPA) se dá através do aumento nos níveis de corticosterona e níveis de adrenocorticotróficos (ACTH) relacionados a predominância de bactérias patogênicas (Sudo, 2016). Todavia o metabolismo microbiano produz a partir de fermentação, ácidos graxos de cadeia curta (AGCC), metabólitos bacterianos neuroprotetivos que podem modular o cérebro e comportamento (John et al., 2014).

A Disbiose é afetada diretamente por comportamento depressivo e ansioso ao aumentar a permeabilidade da barreira intestinal, expondo o organismo a ações de bactérias patogênicas. Um dos mecanismos é a exposição das células do epitélio intestinal ao fator de necrose tumoral alfa (TNF-a), indutor de apoptose celular. A inflamação desencadeada pela disbiose influencia negativamente o sistema nervoso central (SNC) pela regulação de citocinas inflamatórias, com efeitos negativos 
para o cérebro que incluem regulação negativa da via do triptofano para produção de quinurenina, gerando efeitos excitotóxicos e neurotóxicos (Guillemin et al., 2012).

Inflamações que acometem o trato gastrointestinal baixo (TGI) como síndrome do Intestino Irritável (SII), doença de Chronh e colite ulcerativa possuem substancial impacto na qualidade de vida. Além de dores abdominais, cerca de 30\% de indivíduos portadores de SII apresentam desordens psicológicas que incluem ansiedade e depressão. A comunicação bilateral intestino-cérebro levam os fatores psicológicos influenciar a função gastrointestinal induzida por estresse, que resulta na ativação do eixo hipotálamo-hipófise-adrenal (HPA). O eixo HPA entre suas funções, medeia a liberação de hormônios adrenocorticotróficos, que exercem efeitos diretos no trato gastrointestinal, como aumento da permeabilidade intestinal e aumento da secreção de glicocorticosteroides (Gracie et al., 2019).

A microbiota estabilizada produz nível adequado de neurotransmissor estabilizador como gama-aminobutirico (GABA) a parir de glutamato monossódico, norepinefrina, serotonina e dopamina. A adequada composição da microbiota intestinal aumenta a produção de fator neurotrófico derivado do cérebro (BDNF), fator associado a neuroplasticidade, no hipocampo que está intimamente associado a depressão e estresse (Evrensel et al., 2015).

Dentro desse contexto sobre microbiota intestinal surgem algumas pesquisas com espécies ser bactérias focam nos beneficios que que determinadas bactérias podem trazer ao seu hospedeiro. A Bifidobacterium longum e Lactobacillus helveticus mostraram benefícios ao diminuir desconfortos gastrointestinais em pessoas afetadas pelo estresse ao passo que demonstraram melhoras no comportamento emocional e psicológico. Bifidobacterium longum mostrou reduzir a ansiedade e melhorias em resposta ao estresse em indivíduos saudáveis (Allen et al., 2016).

As bactérias Lactobacillus helveticus e Bifidobacterium longum foram precisamente testadas para melhorar o humor, estresse e ansiedade em indivíduos com baixo humor. Romijm et al. (2017), em estudo controlado por placebo, suplementou 79 indivíduos adultos com depressão moderada previamente diagnosticados por Escala de Depressão, Ansiedade e Estresse (DASS-42, inglês) e Inventário Rápido de Sintomatologia Depressiva (QIDS-SR16, inglês). Apesar de biomarcadores sanguíneos como CRP, IL-1 $\beta$, IL-6 e TNF- $\alpha$, não apresentarem mudanças sigficativas, o grupo probiótico demonstrou aumento significativo nos níveis de calciferol (vitamina D), correlacionada a modulação intestinal e processos antinflamatórios.

Em estudo randomizado, duplo-cego e controlado por placebo, Akkasheh et al. (2016), abordou a utilização de probióticos em 40 pacientes diagnosticados com EDM. Durante 8 semanas os pacientes consumiram cápsulas contendo probióticos Lactobacillus acidophilus, Lactobacillus casei e Bifidobacterium longum ou placebo. O grupo probiótico mostrou significativa regressão na pontuação, -5.7, avaliada pela Escala de Depressão Beck (BDI, inglês) contra -1.5 em relação ao placebo. A glutationa mostrou incremento de $1.8 \pm 83.1$ contra $-106.8 \pm 190.7 \mu \mathrm{mol} / \mathrm{L}$ do grupo placebo.

Há uma ampla interação entre o intestino humano entre os nutrientes, microbiota e células hospedeiras. Durante muito tempo considerou-se apenas a via patogênica destes microorganismos haja visto que suas toxinas invadem a mucosa intestinal e se translocam, se disseminam e causam infecções sistêmicas (BUTEL et al., 2014). O ácido gama-aminobutírico (GABA) é importante neurotransmissor inibitório no sistema nervoso central (SNC) e disfunções na sinalização GABA estão ligados para ao comportamento ansioso e depressivo (Foster et al., 2013). Bactérias como Lactobacillus e Bifidobacterium demonstram capacidade de metabolizar glutamato para produzir GABA, influenciando comportamentos semelhantes a ansiedade e depressão, além de alterar a expressão central dos receptores GABA nas principais regiões do cérebro relacionadas ao estresse do sistema nervoso central (Barrett et al., 2012).

De acordo com as informações precitadas faz-se necessário uma abordagem voltada para as novas opções de tratamento substitutivas e ou adjuvantes, visando o tratamento da depressão de forma mais ampla para regressão ou redução de sua sintomatologia. Diante desse cenário a importância da aplicabilidade desse trabalho é justificada e sustentada por dados 
científicos, que mostram a necessidade de intervenções nutricionais visando a modulação da microbiota intestinal através da utilização de probióticos como uma ferramenta no tratamento complementar em desordens depressivas.

Essa pesquisa teve como objetivo geral descrever as contribuições da utilização de probióticos na modulação intestinal como adjuvante no tratamento de sinais e sintomas de depressão. Como objetivos específicos identificar gêneros bacterianos para o tratamento da depressão, identificar doses de probióticos para o tratamento da depressão e identificar suas aplicações clínicas no tratamento da depressão.

\section{Metodologia}

\subsection{Tipo de estudo}

O presente estudo tratara-se de uma pesquisa bibliográfica do tipo revisão integrativa da literatura. Este procedimento foi selecionado por possibilitar a síntese e análise do conhecimento científico já produzido sobre o tema "PROBIÓTICOS NA MODULAÇÃO INTESTINAL COMO ADJUVANTE NO TRATAMENTO DE SINAIS E SINTOMAS DE DEPRESSÃO”

A pesquisa bibliográfica é um estudo elaborado com base em material já produzido, embasado especialmente de livros e artigos científicos, sendo que há também pesquisas produzidas somente de fontes bibliográficas. A abordagem ampla de conteúdos permitida ao pesquisador além daquela que poderia pesquisar diretamente, é a principal vantagem desse tipo de estudo, que se torna de grande importância quando o assunto a ser trabalhado necessita de dados variados (GIL, 2017).

De acordo com Ercole, Melo e Alcoforado (2014), a revisão integrativa de literatura é um método que tem como finalidade sintetizar resultados obtidos em pesquisas de maneira sistemática, ordenada e abrangente, mediante diferentes metodologias. É denominada integrativa, porque fornece informações mais amplas sobre um assunto, constituindo um corpo de conhecimento e podendo ser direcionada para a definição de conceitos, revisão de teorias ou análise metodológica dos estudos. Este método proporciona a combinação de dados da literatura teórica e empírica, proporcionando maior compreensão do tema de interesse. Associação- Microbiota AND Depression AND Probiotic.

\subsection{Identificação do tema e seleção da questão de pesquisa}

A partir do problema de pesquisa "Suplementação de probióticos na modulação intestinal pode ser benéfica no tratamento de sinais e sintomas de depressão?", determinou a construção da estratégia PICO, que representa um acrômio para Paciente/problema (P), Interesse (I), Contexto (Co) OU Comparação(c) e Outcome (O), na qual foi utilizada para a localização dos estudos relevantes, que respondessem à pergunta de pesquisa. Foi realizado a busca de descritores indexados e não indexados: Depressão, Microbiota e Probiótico no idioma inglês. A partir dos descritores em PubMed como mostra o Quadro 1. 
Quadro 1. Elementos da estratégia PICO descritores e palavras-chave utilizados - Caxias, MA, Brasil, 2020.

\begin{tabular}{|c|c|c|c|c|c|}
\hline \multicolumn{2}{|c|}{ Elementos } & \multirow{2}{*}{$\begin{array}{l}\text { Mesh } \\
\text { "depression" } \\
\text { "depressive disroder" }\end{array}$} & \multirow{2}{*}{$\begin{array}{l}\text { Decs } \\
\text { “depressoão" ou } \\
\text { "desordem } \\
\text { depressiva" }\end{array}$} & \multirow{2}{*}{$\begin{array}{l}\begin{array}{l}\text { Outras Bases } \\
\text { de Dados }\end{array} \\
\text { Não se aplica }\end{array}$} & \multirow{2}{*}{$\begin{array}{c}\text { Palavras- chave } \\
\text { Pacientes } \\
\text { depressivos }\end{array}$} \\
\hline $\mathbf{P}$ & $\begin{array}{l}\text { Pacientes } \\
\text { humanos }\end{array}$ & & & & \\
\hline I & $\begin{array}{c}\text { Suplementação } \\
\text { probiótico }\end{array}$ & $\begin{array}{l}\text { "probiotics" } \\
\text { "dietary supplemets" } \\
\text { "probiotical" }\end{array}$ & $\begin{array}{l}\text { "probióticos" } \\
\text { "suplemento } \\
\text { diético" } \\
\text { "probiótico" }\end{array}$ & Não se aplica & $\begin{array}{c}\text { Suplementação } \\
\text { probiótico }\end{array}$ \\
\hline $\mathbf{C}$ & $\begin{array}{l}\text { Comparação } \\
\text { grupo controle }\end{array}$ & Não se aplica & Não se aplica & Não se aplica & Estudo clínico \\
\hline $\mathbf{O}$ & $\begin{array}{l}\text { Regressão } \\
\text { sintomas } \\
\text { depressivos }\end{array}$ & $\begin{array}{l}\text { "depressives" } \\
\text { "regression } \\
\text { psychology" }\end{array}$ & $\begin{array}{l}\text { “depressão" } \\
\text { "regressão } \\
\text { depressive" }\end{array}$ & Não se aplica & Regressão depressão \\
\hline
\end{tabular}

Fonte: Elaboração Própria (2020).

Foram examinados por meio de descritores e palavras-chave as bases de dados PubMed. Os termos utilizados durante a pesquisa foram classificados e combinados nos bancos de dados, resultando em estratégias específicas da base pesquisada.

Quadro 2. Estratégias de busca utilizadas nas bases de dados PUBMED.

\begin{tabular}{|c|c|c|c|c|}
\hline Base de Dado & Estratégia de Busca & Resultados & Filtrados & Selecionados \\
\hline $\begin{array}{c}\text { PubMed (descriptors } \\
\text { MeSH) }\end{array}$ & $\begin{array}{c}\text { Depression AND } \\
\text { Microbiota AND Probiotic }\end{array}$ & 190 & 50 & 12 \\
\hline
\end{tabular}

Fonte: Bases de dados (2020).

\subsection{Estabelecimento dos critérios de inclusão e exclusão}

Após as buscas nas bases de dados utilizando a associação Microbiota AND Depression AND Probiotics, foram encontrados 190 artigos, mediante aplicação dos critérios de inclusão: artigos em inglês, estudo piloto, ensaios clínicos randomizadocontrolado e estudos publicados entre 2010 a 2020 restaram 105. Destes, após a aplicação dos critérios de exclusão, os principais estudos que utilizaram medicamentos antidepressivos associados a probióticos, restaram 50 publicações. Dessas, 12 publicações apresentavam metodologia, temática e objetivos condizentes com os objetivos da pesquisa atual (Figura 1). 
Figura 1. Representação gráfica da aplicação dos critérios de inclusão e exclusão para a associação.

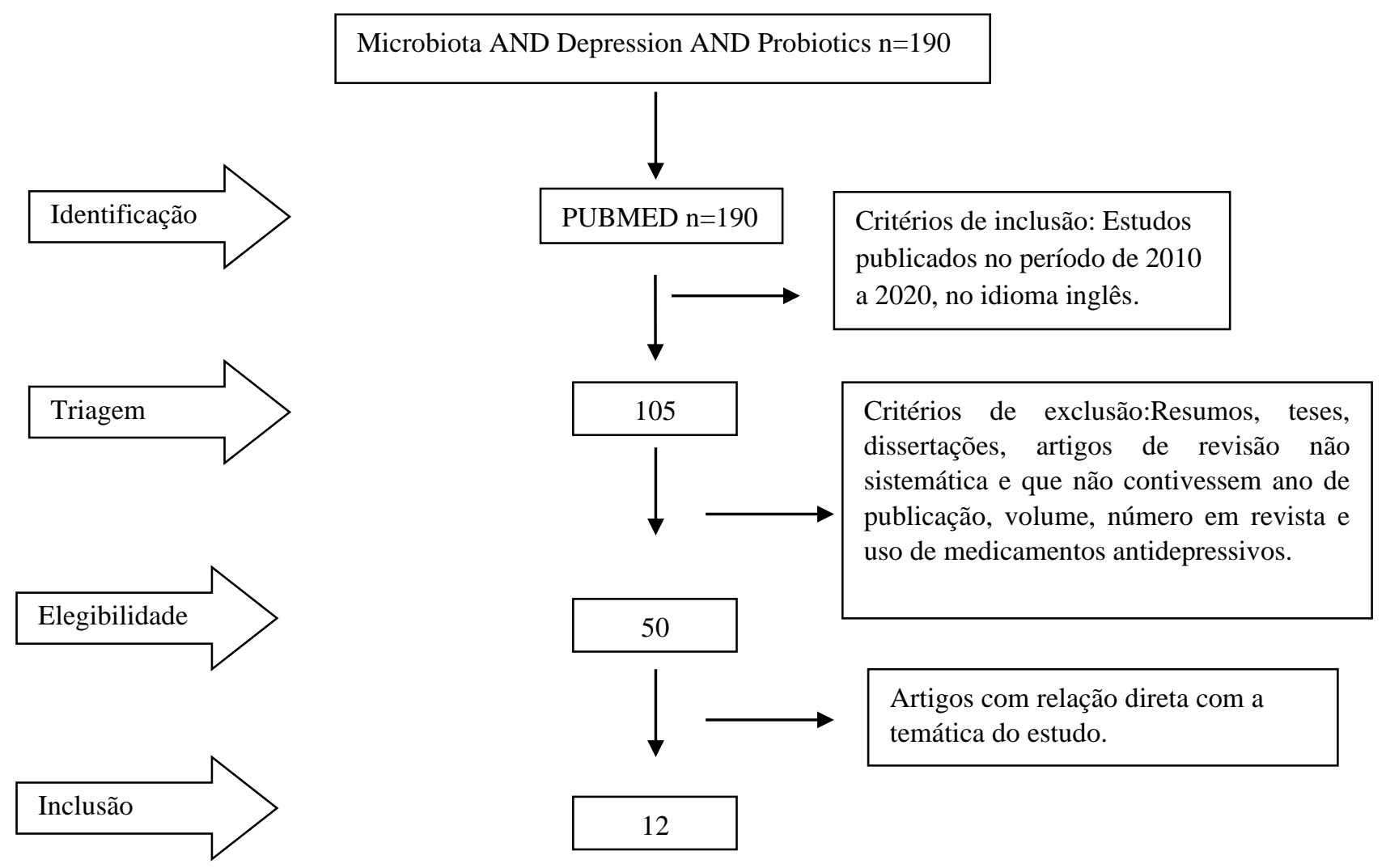

Fonte: Pesquisa direta (2020).

\section{Resultados}

Caraterização dos estudos, segundo o ano de publicação, observou-se uma prevalência de publicações nos anos de 2016 seguidas por 2015 e 2017.

Tabela 1. caracterização dos estudos, segundo o ano de publicação.

\begin{tabular}{|c|c|c|}
\hline Ano da publicação & Número absoluto & $\%$ \\
\hline 2018 & 1 & 7.7 \\
\hline 2017 & 2 & 16.66 \\
\hline 2016 & 4 & 30.76 \\
\hline 2015 & 3 & 23.07 \\
\hline 2014 & 1 & 7.7 \\
\hline 2011 & 1 & 7.7 \\
\hline TOTAL & 12 & $100 \%$ \\
\hline
\end{tabular}

Fonte: Pesquisa em bases de dados (2020).

A Tabela 2 expõe a distribuição dos estudos, conforme o idioma de publicação, observou-se uma predominância de estudos na língua inglesa, correspondendo $100 \%$ dos estudos selecionados. 
Research, Society and Development, v. 10, n. 8, e55910816634, 2021

(CC BY 4.0) | ISSN 2525-3409 | DOI: http://dx.doi.org/10.33448/rsd-v10i8.16634

Tabela 2. Distribuição dos artigos de acordo com o idioma.

\begin{tabular}{lcc}
\hline Idioma & Número absoluto & $\%$ \\
\hline Inglês & 12 & $100 \%$ \\
\hline TOTAL & 12 & $100 \%$ \\
\hline
\end{tabular}

Fonte: Pesquisa em bases de dados (2020).

\subsection{Probióticos e depressão/ansiedade}

Foi avaliado os dados de 922 pacientes com transtornos de depressão/ansiedade. Houve diferenças significativas nas escalas avaliativas entre os 12 diferentes estudos utilizando probióticos.

\subsection{Análise de subgrupo de eficácia probiótica com base em diferentes escalas}

Todos os 12 estudos envolveram escalas diferentes, esses estudos foram categorizados em 8 escalas: BDI, HADS, DASS-21 LEIDS, HAMD, STAI, MADRS e CES-D. 
Research, Society and Development, v. 10, n. 8, e55910816634, 2021

(CC BY 4.0) | ISSN 2525-3409 | DOI: http://dx.doi.org/10.33448/rsd-v10i8.16634

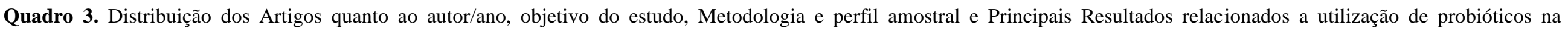
modulação intestinal como adjuvante no tratamento de sinais e sintomas de depressão.

\begin{tabular}{|c|c|c|c|}
\hline Autor (es) /Ano & $\begin{array}{l}\text { Objetivo Do } \\
\text { estudo }\end{array}$ & Metodologia e perfil amostral & Principais Resultados \\
\hline $\begin{array}{l}\text { Muhammed Majeed et al. } \\
\text { (2018). India }\end{array}$ & $\begin{array}{l}\text { Avaliar a segurança e eficácia da cepa } \\
\text { probiótica Bacillus coagulans para TDM em } \\
\text { pacientes com SII. }\end{array}$ & $\begin{array}{l}\text { Ensaio clínico, randomizado, duplo } \\
\text { cego, controlado por placebo. } \\
(\mathrm{n}=40)\end{array}$ & $\begin{array}{l}\text { Pacientes (20-65 anos) diagnosticados com TDM e SII, } \\
\text { suplementados por } 90 \text { dias, apresentaram melhoras no } \\
\text { TDM avaliado por HAM-D, MADRS e CES-D }\end{array}$ \\
\hline $\begin{array}{l}\text { Romijn et al. (2017). Nova } \\
\text { Zelândia }\end{array}$ & $\begin{array}{l}\text { Investigar efeito dos probióticos na melhora } \\
\text { do humor, estresse e ansiedade em uma } \\
\text { amostra selecionada para o baixo humor. } \\
\text { Investigar se a presença ou gravidade dos } \\
\text { sintomas da SII e os níveis de citocinas pró- } \\
\text { inflamatórias, fator, BDNF e outros } \\
\text { marcadores sanguíneos poderiam prever ou } \\
\text { impactar a resposta ao tratamento. }\end{array}$ & $\begin{array}{l}\text { Ensaio clinico randomizado } \\
\text { controlado, duplo cego com placebo. } \\
(\mathrm{n}=79)\end{array}$ & $\begin{array}{l}\text { Indivíduos (adultos }+16 \text { anos) suplementados com } \\
\text { Lactobacillus helveticus e Bifidobacterium longum }(3 \times 109 \\
\text { CFU/g) demonstrou melhoras } \geqslant 60 \% \text { na escala MADRS } \\
\text { em } 8 \text { semanas. }\end{array}$ \\
\hline $\begin{array}{l}\text { Pinto-Sanchez et a } \\
\text { (2017). Canada. }\end{array}$ & $\begin{array}{l}\text { Avaliar os efeitos de Bifidobacterium } \\
\text { longum na ansiedade e depressão em } \\
\text { pacientes com SII. }\end{array}$ & $\begin{array}{l}\text { Ensaio clinico randomizado } \\
\text { controlado, duplo cego com placebo } \\
(\mathrm{n}=44)\end{array}$ & $\begin{array}{l}\text { Adultos } \quad(26-58 \quad \text { anos }) \quad \text { suplementados } \\
\text { Bifidobacterium longum }(1 \times 1010 \text { CFU/g) em indivíduos } \\
\text { com Síndromes do Intestino Irritável }(\mathrm{SII}) \text {, melhorou os } \\
\text { sintomas depressivos e qualidade de vida avaliada por } \\
\text { HADS. }\end{array}$ \\
\hline
\end{tabular}


Research, Society and Development, v. 10, n. 8, e55910816634, 2021

(CC BY 4.0) | ISSN 2525-3409 | DOI: http://dx.doi.org/10.33448/rsd-v10i8.16634

\section{Muhammadi et al. (2016).}

Iran
O objetivo deste estudo foi determinar os efeitos do iogurte probiótico e da suplementação de cápsulas probióticas multiespécies na saúde mental e no eixo hipotálamo-hipófise-adrenal em trabalhadores do setor petroquímico.
Ensaio clinico randomizado controlado, duplo cego com placebo $(\mathrm{n}=45)$

\section{Akkasheh et al. (2016).}

Iran.

\section{Kato-Kataoka, et al (2016). Japão}

Ebrahim et al. (2016). Iran

\section{Steenbergen et \\ al. (2015).}

Holanda
Examinar os efeitos da suplementação de probióticos nos sintomas de depressão, perfis metabólicos, PCR-us e biomarcadores de estresse oxidativo em pacientes com TDM.

Investigar os efeitos do probiótico

Lactobacillus casei, cepa Shirota, sobre a disfunção abdominal, conduzido com estudantes de medicina saudáveis realizando exame nacional.

Este ensaio foi realizado para avaliar os efeitos da ingestão de probióticos na incapacidade, saúde mental e condição metabólica em indivíduos com EM.

Objetivo testar a suplementação probiótica multiespécies podem reduzir a reatividade cognitiva em indivíduos deprimidos.
Ensaio clinico randomizado controlado, duplo cego com placebo $(n=40)$

Duplo cego, controlado por placebo, grupo paralelo $(n=47)$

Ensaio clinico randomizado controlado, duplo cego com placebo. $(n=60)$

Ensaio clinico randomizado controlado, triplo cego com placebo. $(\mathrm{n}=20)$
Voluntários (20-60 anos) suplementados com iogurte (100g/dia) contendo probióticos Lactobacillus acidophilus e Bifidobacterium latis $\left(1 \times 10^{7} \mathrm{CFU} / \mathrm{g}\right)$ ou cápsula de cepas probióticas, Actobacillus casei $\left(3 \times 10^{3} \mathrm{CFU} / \mathrm{g}\right)$, Lactobacillus acidophilus $\left.3 \times 10^{3} \mathrm{CFU} / \mathrm{g}\right)$, Lactobacillus rhamnosus $\left(7 \times 10^{9}\right.$ $\mathrm{CFU} / \mathrm{g})$, Lactobacillus bulgaricus $(5 \times 108 \mathrm{CFU} / \mathrm{g})$,

Bifidobacterium breve $\left(2 \times 10^{10} \mathrm{CFU} / \mathrm{g}\right)$, Bifidobacterium longum $\left(1 \times 10^{9} \mathrm{CFU} / \mathrm{g}\right)$, Streptococcus thermophilus $\left(3 \times 10^{8}\right.$ $\mathrm{CFU} / \mathrm{g}$ ) por 6 semanas, melhoraram significativamente avaliação por DASS-21 e QSG-12

Redução significativa do TDM em pacientes (20-55 anos) suplementados com Lactobacillus acidophilus $\left(2 \times 10^{9} \mathrm{CFU} / \mathrm{g}\right)$, Lactobacillus casei $\left(2 \times 10^{9} \mathrm{CFU} / \mathrm{g}\right)$ e Bifidobacterium bifdum $\left(2 \times 10^{9} \mathrm{CFU} / \mathrm{g}\right)$ por 8 semanas na BDI.

Indivíduos (30 anos) suplementados com Lactobacillus casei Shiroda, em leite fermentado $(100 \mathrm{ml} / \mathrm{dia})$ por 8 semanas foi capaz de prevenir a ansiedade avaliada por VAS

Pacientes com esclerose múltipla, suplementados com Lactobacillus fermentum, Bifidobacterium bifidum,

Lactobacillus acidophilus, Lactobacillus casei, cada cepa a $2 \times 10^{9} \mathrm{CFU} / g$ por 12 semanas, apresentaram redução em ansiedade avaliadas por EDSS

Indivíduos adultos (19.7 anos) suplementados com

Bifidobacterium bifidum, Bifidobacterium

Lactis, Lactobacillus acidophilus, Lactobacillus brevis, Lactobacillus casei, Lactibacillys salivarius, Lactobacillus lactis $\left(2.5 \times 10^{9} \mathrm{CFU} / \mathrm{g}\right)$, apresentaram significativas reduções mensurada por LEIDS. 


\section{Yang et al.
(2015). China \\ Yang et al.
(2015). China}

Objetivo de elucidar o efeito dos

probióticos na melhora da ansiedade e IRC

sérica em pacientes com câncer de laringe antes da cirurgia.

Slykerman et al. (2015). Nova Zelândia

\section{Lorenzo-Zúñiga et} (2014). Espanha

al. Determinar os efeitos relacionados à dose de uma nova combinação de probióticos, na qualidade de vida relacionada à SII.

rhamnosus administrado na gravidez e no pós-parto sobre os sintomas de depressão e ansiedade materna no período pós-parto e
Ensaio clínico,

randomizado, controlado

placebo. $(\mathrm{N}=50)$

Ensaio clínico, duplo

cego, controlado

$(\mathrm{N}=413)$

Ensaio

clínico

multicêntrico, randomizado, duplo-cego, controlado por

placebo.

$(\mathrm{n}=84)$

Investigar a atividade do tipo ansiolítica de formulação probiótica e seus possíveis efeitos na ansiedade, depressão, estresse e estratégias de enfrentamento em voluntários saudáveis.

Pacientes com câncer suplementados com Clostridium

butyricum (420mg), apresentaram melhoras na HAM-D

Mulheres pós púberes com depressão pós parto,

suplementadas com Lactobacillus rhamnosus $\left(6 \times 10^{9} \mathrm{CFU} / \mathrm{g}\right)$ por 1 ano apresentaram redução no STAI-6.

Pacientes (20-70 anos) com SII, suplementados com duas cepas de Lactobacillus plantarum e uma cepa Pediococcus acidilactici em doses alta de $1-3 \times 10^{10} \mathrm{CFU} / \mathrm{g}$ e doses baixas de $3-6 \times 10^{9} \mathrm{CFU} / \mathrm{g}$. Ambos os grupos tratados com altas doses e baixas doses apresentaram melhoras no questionário de VSI sobre a redução da ansiedade e melhoras no IBS-QoL.

Pacientes (18-23 anos) suplementados com pobióticos Lactobacillus helveticus e Bifidobacterium longum $\left(3 \times 10^{9}\right.$ $\mathrm{CFU} / \mathrm{g}$ ) por 30 dias, melhorou o índice na HADS. Na escala HSCL-90 três de seus sub itens avaliados como depressão, somatização e raiva.

Transtorno depressivo maior, TDM; Proteína C-reativa ultra sensível, PCR-us; Escala de Depressão Beck, BDI; por Avaliação de Sintomas 90 (SCL-90-R); Escala Hospitalar de Ansiedade e Depressão, HADS; Escala de Sintomas Hopkins, HSCL-90; Escala de Depressão, Ansiedade e Estresse, DASS-21; Questionário de Saúde Geral, QSG; Leiden Index of Depression Sensitivity, LEIDS; liberador de corticotropina IRC; Avaliação de Depressão de Hamilton, HAM-D; Inventário de Ansiedade Traço-Estado, STAI-6; escala visual analógica, VAS; Esclerose Múltipla, EM; Escala Expandida do Estado de Incapacidade, EDSS; Sensibilidade Visceral, VSI; Síndrome do Intestino Irritável, SII; fator neurotrófico derivado do cérebro, BDNF; Escalas de depressão de Montgomery \& Asberg, MADRS; Irritable Bowel Syndrome Quality of Life, IBS-QoL; Escala de Depressão do Centro de Estudos Epidemiológicos, CES-D; Fonte: Pesquisa direta (2020). 
Research, Society and Development, v. 10, n. 8, e55910816634, 2021

(CC BY 4.0) | ISSN 2525-3409 | DOI: http://dx.doi.org/10.33448/rsd-v10i8.16634

\section{Discussão}

Durante 8 semanas de suplementação de probióticos, Akkasheh et al. (2016), avaliou os pacientes, com base em Escala de Depressão Beck (BDI) do início ao final do estudo juntamente com coleta de amostras sanguíneas. Quando comparada ao grupo placebo, pacientes que receberam intervenções com probióticos, reduziram significativamente a pontuação total na BDI $-5.7 \pm 6.4$ contra placebo de $-1.5 \pm 4.8$, $(\mathrm{P}=0.001)$. Observou -se significativas reduções em níveis de insulina $-2.3 \pm 4.1$ contra placebo de $+2.6 \pm 9.3 \mu \mathrm{IU} / \mathrm{mL},(\mathrm{P}=0.03)$, HOMA-IR $0.6 \pm 1.2$ contra placebo $+0.6 \pm 2.1(\mathrm{P}=0.03), \mathrm{PCR}-$ us $-1138.7 \pm 2274.9$ contra placebo $+188.4 \pm 1455.5 \mathrm{ng} / \mathrm{mL},(\mathrm{P}=0.03)$.

Adicionalmente a glutationa (GSH) apresentou aumento $+1.8 \pm 83.1$ contra placebo $106.8 \pm 190.7 \mu \mathrm{mol} / \mathrm{L}(\mathrm{P}=0.02)$. Melhoras nos níveis de marcadores glicêmicos com suplementação de probióticos é demonstrado em meta análise de Vajihe Akbari et al. (2016) com 13 artigos e 2736 pacientes diabéticos tipo II onde probióticos demonstraram diferenças significativas $(\mathrm{p}<0.05)$ em relacionados a resistência insulínica.

Messaoudi et al. (2011), incluíram através de avaliação por Escala Hospitalar de Ansiedade e Depressão (HADS) e avaliados em exames preliminares por Avaliação de Sintomas 90 (SCL-90-R) e urina. Cada participante recebeu probióticos para 30 dias. As diferenças na escala de 1.5-2 pontos em Escala de Sintomas Hopkins (HSCL-90) foram consideradas significativas. Os resultados obtidos por teste U de Mann-Whitney (MWT) foi favorável ao grupo suplementado com probiótico $\mathrm{z}=1.98$

$(\mathrm{P}<0.05)$ assim como resultados que avaliaram melhoras na subescala HADSAnsiedade para $(\mathrm{MWT}: \mathrm{z}=2 \cdot 19 ; \mathrm{P}=0.03$ e $\mathrm{z}=1.92 ; \mathrm{P}=0.06)$ respectivamente. $\mathrm{O}$ cortisol livre mensurado em urina $(\mathrm{ng} / \mathrm{ml})$ não demonstraram diferenças entre grupo probiótico e placebo, porém foi observado uma tendência de diminuição no grupo probiótico (43.7 (29.2-56.6) em relação a exames de base $(50 \cdot 5(39 \cdot 8-68))$. Posteriormente E. Aizawa et al. (2016) demonstra diferenças na colônicas em pacientes com TDM apresentavam contagens de Bifidobacterium significativamente mais baixas e tendiam a ter contagens mais baixas de Lactobacillus.

Mohammadi et al. (2016), selecionaram previamente trabalhadores de indústria petroquímica avaliados por Questionário de Saúde Geral (QSG) e Escala de Depressão, Ansiedade e Estresse (DASS-21) e avaliações secundárias de quinurenina, triptofano, neuropeptídeo $\mathrm{Y}$, cortisol e hormônio adrenocorticotrófico (ACTH). O grupo iogurte probiótico+placebo apresentou melhoras significativas no QSG-12 (18.0 \pm 1.5 e controle $13.5 \pm 1.9(\mathrm{P}=0.007)$ e grupo capsula probiótica+iogurte convencional $16.9 \pm 1.8$ e controle $9.8 \pm 1.9(\mathrm{P}=0.001)$ e melhoras significativas nas pontuações Escala de Depressão, Ansiedade e Estresse (DASS-21) no grupo iogurte probiótico+placebo (23.3 \pm 3.7 e controle $13.0 \pm 3.7(\mathrm{P}=0.02)$ e cápsula probiótica 18.9 \pm 3.2 e controle $9.4 \pm 4.0(\mathrm{P}=0.006)$. As concentrações de quinurenina e relação quinurenina/triptofano diferiram significativamente entre grupos convencionais de iogurte probiótico e grupo cápsula probiótica $(\mathrm{P}=0,006)$. Estudo de M. A. Carvalho et só (2017) evidência que a via do metabolismo do triptofano para quinurenina contribui para uma menor integridade funcional e estrutural da substância branca e regulando negativamente dopamina (DA) e aumentar vias de glutamato (GLU).

Pinto-Sanchez et al. (2017), selecionou pacientes diagnosticados com SII por critérios Roma III e ansiedade/depressão leve ou moderada por Escala Hospitalar de Ansiedade e Depressão (HADS) e avaliação de amostra sanguínea. Durante seis semanas o grupo suplementado com Bifidobacterium longum demonstrou redução na pontuação de HADS de $78 \%$ na subescala de depressão enquanto grupo placebo demonstrou melhora de 35\% com risco relativo (RR) 1.98; intervalo de confiança (IC) 1.16-3.38; (p=0.04). Secundariamente as melhoras na escala HADS, observou-se melhora na qualidade de vida mas não houve alterações em marcadores inflamatórios como nterleucinas; IL-1 $\beta$, IL-6, IL-8, IL-10, IL12, razão IL-10/IL-12, proteína C-reativa (PCR), fator de necrose tumoral alfa (TNF- $\alpha$ ) e interferon gama (IFN- $\gamma$ ). A redução em marcadores 
Research, Society and Development, v. 10, n. 8, e55910816634, 2021

(CC BY 4.0) | ISSN 2525-3409 | DOI: http://dx.doi.org/10.33448/rsd-v10i8.16634

inflamatórios e melhora em escala de depressão são observadas em estudo de Elaheh Amirani et al. (2020) com reduções significativas em IL-10, fator de inibidor da síntese de citoquinas humanas, PCR e malondialdeído, marcador de estresse oxidativo.

Steenbergen et al. (2015), aplicaram como método de triagem a Mini Entrevista Neuropsiquiátrica Internacional (MINI) para triagem de participantes e randomizados em dois grupos para rever durante quatro semana probiótico ou placebo. Participantes fizeram auto avaliação por LEIDS ao experimentarem disforia leve, conhecida como vulnerabilidade à depressão. Os resultados do grupo suplementado com probióticos apresentou reduções na pontuação por escala LEIDS média do total de pontos obtidos para agressão pré- intervenção foi de $8.68( \pm 0,94)$, para $6.25( \pm 0.98)$, ruminação mental $11.20( \pm 0.90)$ para 8.25 $( \pm 0.93)(\mathrm{p}<0.001)$. Avaliando a pontuação total na escala LEIDS para agressão, controle, desesperança, aversão ao risco, ruminação mental e aceitação, o grupo probiótico apresentou melhoras de $42.75( \pm 3.24)$ para 33.35 ( \pm 3.51$)(\mathrm{p}<0.001)$, reduzindo significativamente a reatividade cognitiva geral à depressão além de um nível moderado de ansiedade para um nível normal.

No estudo de Romijn et al. (2017), recrutaram indivíduos com mais de 16 anos, pelo menos 4 semanas sem uso de medicamentos psiquiátricos e avaliados por Inventário Rápido de Sintomatologia Depressiva (QIDS-SR16) com pontuação o $\geq$ 11 e pela Escala de Depressão, Ansiedade e Estresse (DASS-21) o $\geq 14$ pontos e tiveram sangue coletado para avaliação de biomarcadores. Participantes foram monitorizados a cada duas semanas por questionário online. Durante a avaliação por Escala de depressão de Montgomery \& Asberg (MADRS), o grupo suplementado com probiótico apresentou 23\% de melhora de quadro depressivo e com redução $>60 \%$ na escala MADRS. Não houveram alterações em marcadores inflamatórios como PCR, IL-1 $\beta$, IL-6 ou TNF- $\alpha$, porém foi relacionado positivamente níveis elevados de vitamina D no grupo probiótico com melhoras no humor avaliadas por Inventário Rápido de Sintomatologia Depressiva (QIDS-SR16).

Yang et al. (2015). recrutaram 20 indivíduos com câncer de laringe em pré cirurgia para averiguar a suplementação de (Clostridium butyricum 420mg nas 2 semanas que antecediam a cirurgia é avaliados um dia antes do procedimento. Pacientes foram avaliados por HAM-D e por amostra sanguínea que mensurou fator de liberação de corticotrofina (CRF). Apesar de não influenciar com relevância os níveis de CRF, o grupo suplementado com probiótico reduziu significativamente a pontuação na escala HAM-D de 19.8 para 10.2 (p<0.05). Além disto a suplementação probiótica em pacientes com câncer em tratamento quimioterápico promovem outros benefícios como melhora na qualidade de vida e redução de episódios diarreicos associados a quimioterapia. (Dang Wei et al., 2018)

Em estudo conduzido com grávidas, Slykerman et al. (2017), ao suplementar grávidas em período gestacional de 14 16 semanas com para avaliar depressão e ansiedade durante o período gestacional e depressão pós parto. Gestantes avaliadas por Escala de Depressão Pós-Parto de Edimburg (EPDS) e STAI-6. O grupo probiótico apresentou redução na pontuação depressão 7,7 (SD=5,4), placebo 9,0 (6,0), efeito da amostra -1,2 (95\% CI -2,4-0,1), p=0,035) enquanto ansiedade 12,0 (4,0) e placebo $13,0(\mathrm{SD}=4,3)$, efeito da amostra $-1,1(-1,9,-0,2 \mathrm{p}=0,014)$. Ratificando a internação em grávidas, probióticos mostram-se seguros durante a gestação para prevenção de ansiedade e depressão pré e pós parto e promovem benefícios adicionais aos bebês quanto a redução de eczema e alergias (Pamela D Browne et al., (2019).

Kato-Kataoka, et al. (2016), acompanhou estudantes em fase de exame nacional por oito semanas com consumo de Lactobacillus casei, subespécie Shirota consumidos com leite fermentado e sua influência sobre composição fecal, estado psicológico, biomarcadores de estresse e composição de microbiota analisada por 16S rRNA. A avalição por VAS demonstrou que o grupo suplementado com probiótico apresentou menor sensação de estresse, menor nível de cortisol mensurado por saliva, não apresentaram eventos de disfunção gastrointestinal e apresentaram melhoras nos níveis celulares de células NK em $17.7 \pm 1.3$ (células/ml), pré exame $16.3 \pm 1.5$ e pós exame de $11.2 \pm 0.9$ quando comparado a placebo $10.2 \pm 0.9$ (p < 0.01 ).Por 
análise unidade taxonômica operacional (OTU), A Naseribafrouei et al. (2014) previamente constata correlações entre depressão e microbiota fecal em 37 pacientes com relação Bacteroidetes $(\mathrm{p}=0.05)$ e um clado para Oscillibacter, e um clado dentro de Alistipes, com associação significativa para depressão ( $p=0,03$ e 0,01 , respectivamente).

Em estudo conduzido em portadores de esclerose múltipla, Ebrahim et al. (2016), averiguaram a suplementação de probióticos sobre a incapacidade mental desses. A média de pontos para grupo probiótico e placebo em EDSS foi de 2.3, 2.0, 0-4.5 e 2.2, 2.5,0-4.0 respectivamente e quando comparados após período de intervenção, na escala de EDSS o grupo probiótico melhorou ( $-0.3 \pm 0.6$ e placebo $0.1 \pm 0.3, \mathrm{P}=0.001)$, na escala Escala de Depressão Beck ( $-5.6 \pm 4.9$ e placebo -1.1 $\pm 3.4, \mathrm{P}<0.001)$ Questionário de Saúde Geral $(-9.1 \pm 6.2$ e placebo $-2.6 \pm 6.4, \mathrm{P}<0.001)$ e para; Escala de Depressão, Ansiedade e Estresse (DASS) $(-16.5 \pm 12.9$ vs. $-6.2 \pm 11.0, \mathrm{P}=0.001)$. Melhoras significativas $(\mathrm{p}<0.001)$ afetaram positivamente níveis de insulina, HOMA-IR, HOMA-B e índice qualitativo de verificação da sensibilidade à insulina (QUIKI).

A segurança de probióticos na esclerose múltipla foi evidenciada por Mehran Rahimlou et al. (2020) ao melhorar de forma significativa sobre Questionário de Saúde Geral $(-5.31 \pm 4.62$ contra placebo $-1.81 \pm 4.23(p=0.002))$, Escala de Depressão Beck (-4.81 \pm 0.79 contra placebo $-1.90 \pm 0.96$; ( $\mathrm{p}=0.001))$ e índice de avaliação de dor (PRI) $(-3.81 \pm 6.56$ vs. 0.24 \pm 5.44; $(\mathrm{p}=0.007)$ ). A intervenção aumentou de forma significativa o Fator Neurotrófico Derivado do Cérebro (BDN) e reduziu de forma significativa IL-6 ( $\mathrm{p}<0.001)$.

Estudo conduzido por Lorenzo-Zúñiga et al. (2014), com indivíduos com SII demonstrou os efeitos dos probióticos sobre Irritable Bowel Syndrome Quality of Life (IBS-QoL). Dois grupos foram suplementados com altas doses e baixas doses de probióticos por 6 semanas apresentaram melhoras no IBS-QoL de $18 \pm 3$ e $22 \pm 4$ nos respectivos grupos alta dose e baixa dose, enquanto placebo melhora de $9 \pm 3$. A avaliação por VSI demonstrou que ambos os grupos probióticos apresentaram melhoras nas pontuações de $10 \pm 2$ e $14 \pm 2$ para grupo baixa dose e alta dose respectivamente. Posteriormente no estudo de Anna Lyra et al. (2017), conduzido com 340 portadores de SII por 12 semanas utilizando probiótico alta dose e baixa dose melhorou a escala de pontuações avaliada por IBS-QoL com reduções de 52.1(10.7) para 21.9(20.6) com SD -31.2 (21.9) e 53.6 (10.9) para 24.4 (19.4) com SD -29.4 (17.9), respectivamente.

Muhammed Majeed et al. (2018), diagnosticou indivíduos com TDM portadores de SII. Pacientes suplementados com probiótico durante noventa dias. Utilizando diferentes escalas para mensurar TDM, pacientes apresentaram melhoras significativas ao final do estudo por escalas avaliativas: HAM-D $5.9 \pm 4.88$ (início estudo $13.6 \pm 4.41(\mathrm{p} \leq 0.001)$; MADRS $6.0 \pm 5.79$ (início do estudo 16.3 $\pm 5.40, \mathrm{p} \leq 0.001$ ), Escala de Depressão do Centro de Estudos Epidemiológicos (CES-D) 8.0 \pm 6.17 (início do estudo $19.1 \pm 5.25, \mathrm{p} \leq 0.001$ ). Na avaliação da SII com IBS-QoL a avaliação final demonstra diferença significativa $56.1 \pm 31.26$ (início do estudo $106.4 \pm 23.44, \mathrm{p} \leq 0.001$ ).

No Gráfico 1 foram compilados os resultados alcançados pelos estudos em diferentes escalas psicométricas quanto a regressão de suas pontuações em seus respectivos estudos com valores avaliados inicialmente e ao final das in terveções.

A forma gráfica dos resultados demonstra a tendência de regressão dos sinais e sintomas depressivos/ansiosos das diferentes cepas probióticas utilizadas, multiespécies ou espécie única, no decorrer do intervalo das intervenções. 
Gráfico 1. Relação intervenção probióticos e regressão na pontuação das escalas psicométricas por tempo de intervenção.

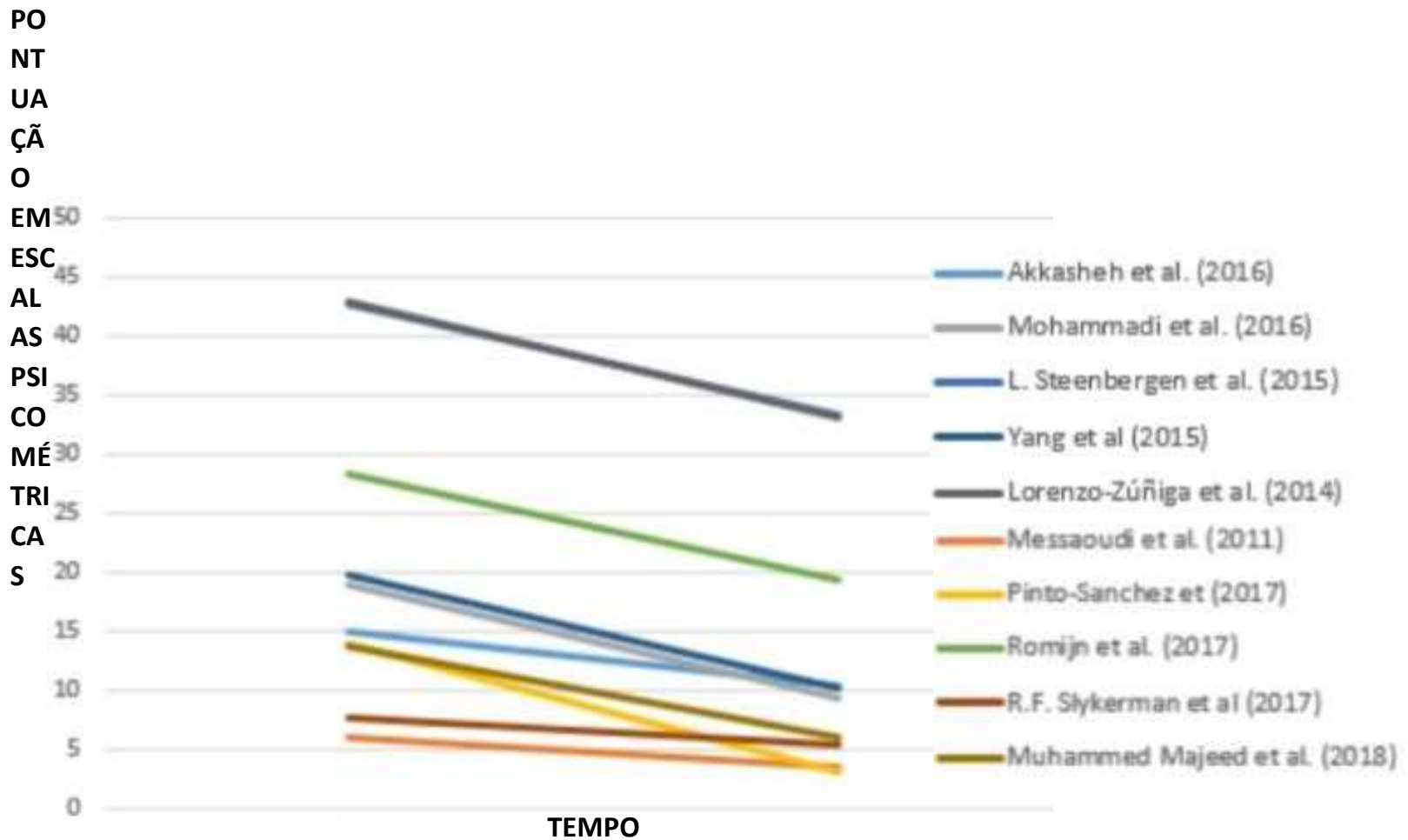

Gráfico 1: Demonstração gráfica da regressão alcançadas ao final das intervenções com grupos probióticos ao longo dos estudos em comparação com avaliações iniciais. O tempo médio de 8 semanas

Fonte: Pesquisa direta (2020).

\section{Conclusão}

Conforme estudos analisados em quadro resumo, evidencia a influência do microbiota intestinal sobre o comportamento humoral de forma a desencadear, amenizar ou a partir de intervenções moduladoras por probióticos atuar na prevenção e tratamento da depressão e transtornos ansiosos. Os estudos elencados, demonstram a influência da microbiota intestinal extra órgão e com influência sistêmica.

A utilização de cepas probióticas mostram-se seguras para a modulação intestinal em pacientes com quadros clínicos de sintomatologia depressiva e ansiosa mesmo apresentando outras comorbidades. As cepas probióticas identificadas com ação psicobiótica, englobam gênero Lactobacillus e Bifidobacterium, podendo ser combinadas em subespécies de seus gêneros, com doses médias de $1 \times 10^{9} \mathrm{CFU} / \mathrm{g}$ e $1 \times 10^{10} \mathrm{CFU} / \mathrm{g}$ por um tempo equivalente a 8 semanas.

A suplementação de probiótico é benéfica para o comportamento e cognição ao modular atividade neural em regiões do cérebro relacionadas ao humor e comportamento. Os resultados deste estudo permitem a construção de conhecimento acerca de doenças psiquiátricas e sobre o manejo no tratamento de indivíduos portadores destas enfermidades.

\section{Referências}

Akbari, M. J., et al. The effects of probiotics on mental health and hypothalamicpituitary-adrenal axis: A randomized, double-blind, placebo-controlled trial in petrochemical workers. Nutr Neurosci. 9, 387-395

Akkasheh, G. K. J., et al. Clinical and metabolic response to probiotic administration in patients with major depressive disorder: a randomized, double-blind, placebocontrolled trial. Nutrition, 32, 315-20 
Research, Society and Development, v. 10, n. 8, e55910816634, 2021

(CC BY 4.0) | ISSN 2525-3409 | DOI: http://dx.doi.org/10.33448/rsd-v10i8.16634

Allen, A. H., et al. Bifidobacterium longum 1714 as a translational psychobiotic: modulation of stress, electrophysiology and neurocognition in healthy volunteers. Transl Psychiatry, 6, 939

Amirani, E., Milajerdi, A, Mirzaei, H., Jamilian, H., Mansournia, M. A., \& Hallajzadeh, J. The effects of probiotic supplementation on mental health, biomarkers of inflammation and oxidative stress in patients with psychiatric disorders: A systematic review and meta-analysis of randomized controlled trials. Complement Ther Med, 49, 102361

Barrett, E., et al. Gamma-Aminobutyric acid productio bacteria from the human intestine. J. Appl. Microbiol, 13, 411-417

Bhandari, et al. Association of Inflammatory Bowel Disease, (IBD) with depressive symptoms in the United States population and independent predictors of depressive symptoms in an IBD population: a NHANES study. Gut Liver, 11,512-9

Bromet, E. A., et al. Cross-national epidemiology of DSM-IV major depressive episode. BMC Med, 9, 90

Ebrahim, K. O. P., et al. Clinical and metabolic response to probiotic supplementation in patients with multiple sclerosis: A randomized, double-blind, placebo-controlled trial. Clin Nutr, 5, 1245-1249

Emiko, A., Hirokazu, T., Takashi, A., Takuya. T. Possible association of Bifidobacterium and Lactobacillus in the gut microbiota of patients with major depressive disorder. Journal of Affective Disorders, 202 254-257

Ercole, F. F.; Melo, L. S.; Alcoforado, C. L. G. C. Revisão Integrativa versus Revisão Sistemática. Rev Min Enferm. 8, 1-260

Gelenberg. A. A. review of the current guidelines for depression treatment. J Clin Psychiatry, 7, e15

Gil, A.C. Métodos e técnicas de pesquisa social. Ediitora Atlas SA

Gracie, P. H. D. et al. The influence of the brain-gut axis in inflammatory bowel disease and possible implications for treatment. The Lancet Gastroenterology \& Hepatology

Guillemin, G. J. Quinolinic acid, the inescapable neurotoxin. FEBS J, 279, 1356-65

John, et al. More than a Gut Feeling: The Microbiota Regulates Neurodevelopment and Behavior. Neuropsychopharmacology Reviews, 40, 241-242

Kato-Kataoka, K. A., et al. Fermented Milk Containing Lactobacillus casei Strain Shirota Preserves the Diversity of the Gut Microbiota and Relieves Abdominal Dysfunction in Healthy Medical Students Exposed to Academic Stress. Appl Environ Microbiol, 12, 3649-58.6

Kwak, M. J., et al. Evolutionary architecture of the infant-adapted group of Bifidobacterium species associated with the probiotic function. Syst Appl Microbiol, 39, 429-39

Lorenzo-Zúñiga, V. E. L., et al. A new combination of probiotics, improves irritable bowel syndrome-related quality of life. World J Gastroenterol, 26, 870916

Lyra, A., et al. Irritable bowel syndrome symptom severity improves equally with probiotic and placebo. World J Gastroenterol, 48, 10631-10642

Michelle, S. et al Metabolismo do triptofano em transtornos mentais: um enfoque em esquizofrenia. VITTALLE - Revista de Ciências da Saúde, 2, 44-56

Ministério da Saúde. Artigo Saúde Mental. Disponível em: https://antigo.saude.gov.br/saude-de-a-z/depressao/851-saude-mental. Acessado 20/10/2020

Naseribafrouei A, Hestad K, Avershina E, Sekelja M, Linløkken A, Wilson R, Rudi K. Correlation between the human fecal microbiota and depression. Neurogastroenterol Motil, 8, 1155-62

Rahimlou M, Hosseini SA, Majdinasab N, Haghighizadeh MH, Husain D. Effects of long-term administration of Multi-Strain Probiotic on circulating levels of BDNF, NGF, IL-6 and mental health in patients with multiple sclerosis: a randomized, double-blind, placebo-controlled trial. Nutr Neurosci, 5, 1-12

Sandhu K.V, et al. Feeding the microbiota-gut-brain axis: Diet, microbiome, and neuropsychiatry. Translational Research, 179, 223-244

Steenbergen, R. S., et al. A randomized controlled trial to test the effect of multispecies probiotics on cognitive reactivity to sad mood. Brain Behav Immun, 4, $8258-64$

Sudo N. Stress and gut microbiota: does postnatal microbial colonization programs the hypothalamic-pituitary-adrenal system for stress response? International Congress Series, Elsevier, 12, 350-354

Vajihe Akbari, Fatemeh Hendijani.Effects of probiotic supplementation in patients with type 2 diabetes: systematic review and meta-analysis. Nutr Ver, 12, 774-784

Wei, D. H Probiotics for the prevention or treatment of chemotherapy- or radiotherapyrelated diarrhoea in people with cancer. Cochrane Database Syst Rev.Aug, 8,8

World H (2017). Organization. Depression Fact https://www.who.int/news-room/fact-sheets/detail/depression.

Yang H, Z. X, et al. Probiotics reduce psychological stress in patients before laryngeal cancer surgery. Asia Pac J Ciln Oncol. 12(1), e92-96. 